\title{
Postoperative mortality in the COVID-positive hip fracture patient, a systematic review and meta-analysis
}

\author{
Alexander Isla ${ }^{1}$ (D) David Landy ${ }^{1}$ (D) $\cdot$ Robert Teasdall $^{1}$ (D) $\cdot$ Peter Mittwede $^{2}$ D $\cdot$ Ashley Albano $^{1}$ (D) Paul Tornetta III ${ }^{3}$ (D) \\ Mohit Bhandari ${ }^{4}$ (D) Arun Aneja ${ }^{1}$ (D)
}

Received: 2 January 2022 / Accepted: 4 February 2022 / Published online: 23 February 2022

(c) The Author(s), under exclusive licence to Springer-Verlag France SAS, part of Springer Nature 2022

\begin{abstract}
Purpose The extent to which concomitant COVID-19 infection increases short-term mortality following hip fracture is not fully understood. A systemic review and meta-analysis of COVID-19 positive hip fracture patients (CPHFPs) undergoing surgery was conducted to explore the association of COVID-19 with short-term mortality.

Methods Review of the literature identified reports of short-term 30-day postoperative mortality in CPHFPs. For studies including a contemporary control group of COVID-19 negative patients, odds ratios of the association between COVID-19 infection and short-term mortality were calculated. Short-term mortality and the association between COVID-19 infection and short-term mortality were meta-analyzed and stratified by hospital screening type using random effects models.

Results Seventeen reports were identified. The short-term mortality in CPHFPs was 34\% (95\% C.I., 30-39\%). Short-term mortality differed slightly across studies that screened all patients, 30\% (95\% C.I., 22-39\%), compared to studies that conditionally screened patients, 36\% (95\% C.I., 31-42\%), $(P=0.22)$. The association between COVID-19 infection and short-term mortality produced an odds ratio of 7.16 (95\% C.I., 4.99-10.27), and this was lower for studies that screened all patients, 4.08 (95\% C.I., 2.31-7.22), compared to studies that conditionally screened patients, 8.32 (95\% C.I., 5.68-12.18), $(P=0.04)$. Conclusion CPHFPs have a short-term mortality rate of $34 \%$. The odds ratio of short-term mortality was significantly higher in studies that screened patients conditionally than in studies that screened all hip fracture patients. This suggests mortality prognostication should consider how COVID-19 infection was identified as asymptomatic patients may fare slightly better.
\end{abstract}

Keywords COVID-19 $\cdot$ SARS-CoV-2 $\cdot$ Hip fracture $\cdot$ Mortality

\section{Introduction}

Coronavirus disease 2019 (COVID-19), caused by the novel severe acute respiratory syndrome coronavirus 2 (SARS$\mathrm{CoV}-2)$, is an international public health emergency. As of December 11, 2020, over 69.6 million cases and approximately 1.58 million deaths have been reported worldwide [1]. Policies to limit its spread have included physical

Arun Aneja

aan247@uky.edu

1 Department of Orthopaedic Surgery, University of Kentucky, 740 South Limestone, Suite K413, Lexington, KY 40536-0284, USA

2 University of Pittsburgh, Pittsburgh, PA, USA

3 Boston University Medical Center, Boston, MA, USA

4 McMaster University, Hamilton, ON, Canada distancing and lockdown measures. The current literature identifies certain groups at high risk of poor COVID-19 outcomes, but data are lacking for some groups, particularly COVID-positive hip fracture patients (CPHFPs).

Hip fractures are the most common traumatic fracture [2]. In the USA alone, they account for 300,000 annual hospitalizations in patients $\geq 65$ [3]. Emerging data shows that during the COVID-19 pandemic, hip fracture incidence has not significantly decreased [4]. This may be attributable to mechanism: $95 \%$ of hip fractures result from low-energy traumas mostly occurring in the home [3, 4]. A recent systematic review of 984 hip fracture patients managed during the COVID-19 pandemic revealed a COVID-19 prevalence of 9\% [2]. Patients who sustain hip fractures are often elderly with a high comorbidity burden, increasing risk of poor COVID-19 outcomes [5-12]. Overlapping inflammatory responses between orthopedic injury and SARS-CoV-2 infection may also contribute 
to poor outcomes [13-15]. Several studies have shown COVID-positive status in hip fracture patients to be associated with increased 30-day mortality [2, 12]. However, the current literature is limited to a small number of retrospective studies conducted during the early phase of the pandemic. The clinical pathways adopted for patient care among these early studies differed considerably. Some of these differences were a result of necessity secondary to limited availability of diagnostic tests during the healthcare crisis created by SARS-CoV-2. Screening capacity in particular varied between medical centers, enabling some to screen all admissions and some to screen only select admissions. Furthermore, it is unknown whether mortality outcomes differed by COVID-19 screening protocol. Given the prevalence and susceptibility of CPHFPs, it is important to understand their surgical outcomes so that providers may more accurately risk stratify and counsel patients and family members. Therefore, the primary aims of this study were to systematically review and meta-analyze: (1) short-term postoperative (30-day) mortality rates in CPHFPs and (2) to explore the association of COVIDpositive status with short-term postoperative hip fracture mortality. In both analyses, we explored effects by screening protocol to understand whether the method of patient identification affected mortality prognostication.

\section{Materials and methods}

The present systematic review was conducted in accordance with Preferred Reporting Items for Systematic Reviews and Meta-Analyses (PRISMA) guidelines [16].

\section{Search strategy}

To identify articles of interest, PubMed, Cochrane Central Database, and EMBASE were searched by two authors independently with "covid AND fracture" with no search filters. Databases were last accessed December 8, 2020. Review of selected article bibliographies was also performed.

\section{Eligibility criteria}

Articles included were those that (1) reported on $\geq 10$ CPHFPs, (2) reported a primary outcome of mortality, (3) were available in English, and (4) were available in full-text. Articles excluded were those that (1) evaluated non-original data, (2) did not evaluate our target population (e.g., pediatric studies and non-human studies), and (3) evaluated only COVID-negative or COVID-unknown patients.

\section{Quality assessment}

Risk of bias assessment for individual articles was performed using the Methodological Index for Non-Randomized Studies (MINORS) criteria [17].

\section{Data extraction}

Data extraction on systematic review of qualifying articles was performed by multiple authors and included study design, study location, total number of hip fracture patients (hip fracture as defined by OTA type 31 fracture) [18], operative versus conservative management, COVID-19 screening protocol, COVID-19 screening method, time to surgery, age, follow-up period, clinical presentation, and mortality. We dichotomized COVID-19 screening protocols as either "all patients screened" or "patients screened conditionally." Regarding clinical presentation, we systematically reviewed for COVID-19 status, time of positive COVID-19 screen (on or after admission), and symptomatology on admission. For the purposes of the present review, patients who recorded only negative COVID-19 screening results for the duration of the study, or who were not tested due to lack of suspicion, were considered COVID-negative. Patients who recorded positive COVID-19 screening results or who were reported to have clinical pictures attributable to COVID-19 alongside absent screening results (presumed positive patients) were considered COVID-positive. We only extracted comparison group data on operatively treated COVID-negative hip fracture patients if treated during the same time period as the study group (CPHFPs). Data reported on historical cohorts, nonoperative COVID-positive or negative hip fractures, or non-hip fractures were ignored. The primary outcome of interest in this review was short-term postoperative mortality.

\section{Analysis}

Due to methodological and reporting differences, short-term mortality rates in operatively managed CPHFPs, expressed as a proportion, and associations between COVID-19 status and mortality at the study level, expressed as an odds ratio of mortality, were meta-analyzed using a random effects model. An overall estimate for each outcome and estimates stratified by screening approach were obtained with stratified estimates compared using the Q test. All sub-group analyses were pre-specified. The decision to use a more conservative random effects model was made independent of empirical estimates of heterogeneity which were assessed using $I^{2}$ and its $95 \%$ confidence interval. Confidence intervals at the $95 \%$ level were calculated for all meta-effect estimates. Funnel 
plots were used to visually inspect for evidence of publication bias for both short-term mortality rates and the association between COVID-19 positive status and short-term mortality. No adjustment was made for multiple hypothesis testing. An alpha threshold of 0.05 was used to define statistical significance. All analyses were performed using $\mathrm{R}$ 3.3.1 (Microsoft, Seattle, WA).

\section{Results}

\section{Search results}

Our search returned 253 articles on PubMed published from December 2019 to December 2020, one article on Cochrane published in October 2018, and 308 articles on EMBASE all published in 2020. After discarding duplicates, screening for inclusion criteria, and applying exclusion criteria, 19 articles were selected for qualitative analysis [7-11, 19-32] and 17 for quantitative analysis (Fig. 1) [7-11, 19-29, 31]. Review of selected bibliographies did not yield additional articles. Studies excluded for reporting on $<10$ CPHFPs included seven single patient case studies and 15 case series, constituting 63 excluded CPHFPs.

\section{Included articles}

Study characteristics are summarized in Table 1. Fourteen retrospective [7, 9-11, 19, 21, 22, 24-28, 30, 32] and four prospective studies $[8,20,29,31]$ were identified. Study design was not specified for one article [23]. Fourteen studies reported data on a contemporary COVID-negative hip fracture group [8-11, 19, 24-32]. All 19 studies combined for a sample size of 3001 hip fracture patients with a mean age of 81.4 (range 41 to $1-101$ ) years [7-11, 19-32]. Six studies reported only on operative patients $[10,21,24,27$,

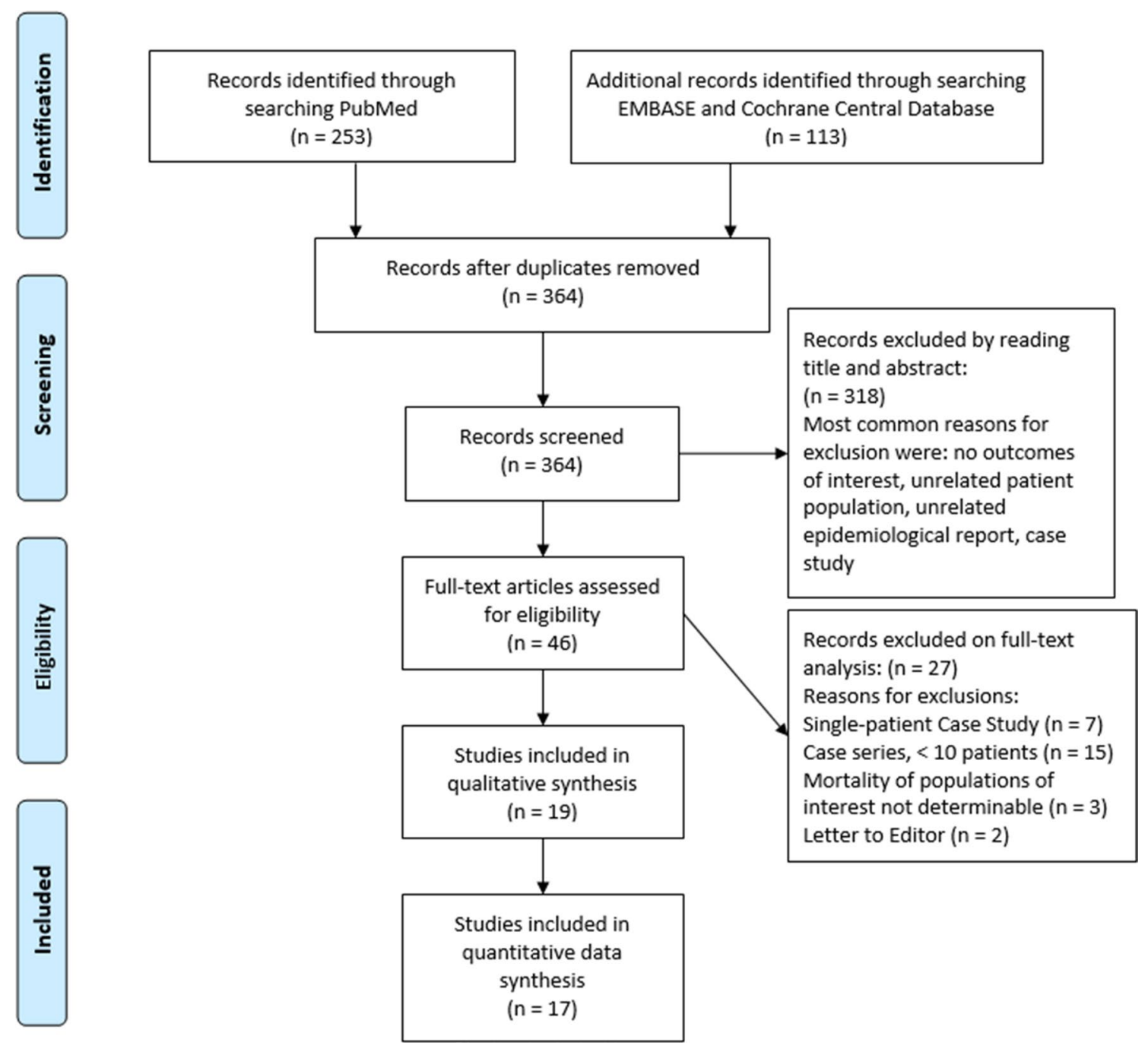

Fig. 1 Prisma flowchart 
Table 1 Study characteristics

\begin{tabular}{|c|c|c|c|c|c|c|c|}
\hline Study & Location & Design & $\begin{array}{l}\text { Total no. of hip } \\
\text { fracture patients }\end{array}$ & $\begin{array}{l}\text { Screening pro- } \\
\text { tocol }\end{array}$ & $\begin{array}{l}\text { Mean delay } \\
\text { to surgery } \\
\text { (h) }\end{array}$ & $\begin{array}{l}\text { Age (range) } \\
\text { (year) }\end{array}$ & Follow-up (days) \\
\hline Arafa et al. [19] & United Kingdom & Retrospective & 97 & Conditional & 28.3 & 83.7 (60 to 99$)$ & 30 \\
\hline $\begin{array}{l}\text { Catellani et al. } \\
{[20]}\end{array}$ & Italy & Prospective & 16 & All Patients & Not reported & 84.3 (74 to 90$)$ & Inpatient \\
\hline Cheung et al. [21] & United States & Retrospective & 10 & All Patients & Not reported & 79.9 (67 to 90$)$ & Inpatient \\
\hline De et al. [22] & United Kingdom & Retrospective & 34 & Conditional & 49.6 & 85.9 & 30 \\
\hline Dupley et al. [7] & United Kingdom & Retrospective & 64 & Conditional & Not reported & 83 (46 to 100$)$ & 45 \\
\hline Egol et al. [8] & United States & Prospective & 138 & Conditional & 33.6 & 83 & 30 \\
\hline Hall et al. [9] & United Kingdom & Retrospective & 317 & Conditional & Not reported & 80.7 (50 to 101$)$ & 30 (minimum) \\
\hline Jannelli et al. [23] & Italy & Not Specified $^{\mathrm{a}}$ & 10 & Conditional & Not reported & 85.6 (77 to 94$)$ & 30 \\
\hline $\begin{array}{l}\text { Karayiannis et al. } \\
\text { [24] }\end{array}$ & United Kingdom & Retrospective & 203 & Not Specified & Not reported & 81.3 (49 to 99$)$ & 30 \\
\hline Kayani et al. [10] & United Kingdom & Retrospective & 422 & All Patients & Not reported & 72.5 & 30 (minimum) \\
\hline LeBrun et al. [11] & United States & Retrospective & 59 & Conditional & 22.8 & 85 (65 to 100$)$ & Inpatient \\
\hline Macey et al. [25] & United Kingdom & Retrospective & 76 & Conditional & 27 & 83 & 30 \\
\hline $\begin{array}{l}\text { Mamarelis et al. } \\
\text { [26] }\end{array}$ & United Kingdom & Retrospective & 37 & All Patients & 33.9 & 80.3 (47 to 99$)$ & 30 \\
\hline $\begin{array}{l}\text { Maniscalco et al. } \\
\text { [27] }\end{array}$ & Italy & Retrospective & 121 & Conditional & Not reported & 81.8 (41 to 99$)$ & 21 \\
\hline Munoz et al. [28] & Spain & Retrospective & 136 & Conditional & 57.6 & 85.3 (65 to 101$)$ & 14 \\
\hline Narang et al. [29] & United Kingdom & Prospective & 682 & Conditional & Not reported & 83.4 & 30 \\
\hline $\begin{array}{l}\text { Rasidovic et al. } \\
\text { [30] }\end{array}$ & United Kingdom & Mixed $^{\mathrm{b}}$ & $404^{c}$ & Conditional & 35.0 & 83.5 & 30 \\
\hline Thakrar et al. [31] & United Kingdom & Prospective & 43 & Conditional & 51.2 & 81.6 (54 to 100$)$ & 30 \\
\hline Ward et al. [32] & United Kingdom & Retrospective & 132 & All Patients & Not reported & 82.1 (49 to 100$)$ & 30 \\
\hline
\end{tabular}

${ }^{a}$ Not specified as retrospective nor prospective

${ }^{b}$ Multicenter trial wherein some centers analyzed data retrospectively and some prospectively

c3/404 patients were missing management data and were excluded from further analysis

$29,31]$, while the remaining 13 studies reported on both operative and nonoperative patients $[7-9,11,19,20,22$, $23,25,26,28,30,32]$. Five studies reported screening all admitted patients for COVID-19 [10, 20, 21, 26, 32], and 13 studies reported conditional COVID-19 screening [7-9, 11, 19, 22, 23, 25, 27-31]. Screening protocol was not specified for one article [24]. While screening methods for COVID-19 patients varied, all studies employed realtime reverse transcriptase polymerase chain reaction (rRTPCR) assays of respiratory swabs for some or all COVID-19 screenings [7-11, 19-32]; some studies used imaging and/ or clinical judgment instead of (or to supplement) rRT-PCR for diagnosis $[8,11,19,20,23,24,26,27,30,32]$. Data on time of positive COVID-19 screen and symptomatology on admission were variably reported; thus, it was not summarized herein. The quality of included articles was moderate (see Table, Supplemental Content 1, which contains full MINORS scoring). The average MINORS scores were 9.8 and 18.6 for non-comparative and comparative studies, respectively.

\section{Outcomes}

Table 2 summarizes patient mortality for each article stratified by management and COVID-19 status. The meta-effect estimate for short-term mortality in operatively managed CPHFPs $(n=466)$ was $34 \%$ (95\% C.I., 30-39\%) (Fig. 2). The $I^{2}$ heterogeneity statistic was $0 \%$ (95\% C.I., $\left.0-46.5 \%\right)$. While there were a limited number of studies within each screening group, the short-term mortality in studies that screened all patients $(n=4 ; 113$ patients) was 30\% (95\% C.I., $22 \%-39 \%$ ) compared to $36 \%$ (95\% C.I., $31-42 \%$ ) in studies that screened patients conditionally $(n=12 ; 332$ patients), though this difference was not statistically significant $(P=0.22)$.

For studies that reported both COVID-positive and negative patients, the meta-effect estimate for the association between COVID-19 positive status and short-term mortality in operatively managed hip fracture patients was an odds ratio of 7.16 (95\% C.I., 4.99-10.27) (Fig. 3). The $I^{2}$ heterogeneity statistic was $13 \%$ (95\% C.I., $0-54 \%)$. The 
Table 2 Mortality by management and COVID-19 status

\begin{tabular}{|c|c|c|c|c|c|c|c|c|}
\hline \multirow[t]{2}{*}{ Study } & \multicolumn{5}{|c|}{ Operative cohort } & \multicolumn{3}{|c|}{ Nonoperative cohort } \\
\hline & Total & COVID + & $\begin{array}{l}\text { COVID + Mortal- } \\
\text { ity }(\%)\end{array}$ & COVID- & $\begin{array}{l}\text { COVID- Mor- } \\
\text { tality (\%) }\end{array}$ & Total & COVID+ & COVID- \\
\hline Arafa et al. [19] & 94 & 17 & $29.41 \%$ & 77 & $7.79 \%$ & 3 & 2 & 1 \\
\hline Catellani et al. [20] & 13 & 13 & $30.77 \%$ & 0 & & 3 & 3 & 0 \\
\hline Cheung et al. [21] & 10 & 10 & $10.00 \%$ & 0 & & 0 & 0 & 0 \\
\hline De et al. [22] & 33 & 33 & $39.39 \%$ & 0 & & 1 & 1 & 0 \\
\hline Dupley et al. [7] & 58 & 58 & $44.83 \%$ & 0 & & 6 & 6 & 0 \\
\hline Egol et al. [8] & 134 & 27 & $33.33 \%$ & 107 & $5.61 \%$ & 4 & 4 & 0 \\
\hline Hall et al. [9] & 303 & 25 & $28.00 \%$ & 278 & $6.83 \%$ & 14 & 2 & 12 \\
\hline Jannelli et al. [23] & 8 & 8 & $25.00 \%$ & 0 & & 2 & 2 & 0 \\
\hline Karayiannis et al. [24] & 203 & 21 & $19.05 \%$ & 182 & $1.65 \%$ & 0 & 0 & 0 \\
\hline Kayani et al. [10] & 422 & 82 & $30.49 \%$ & 340 & $10.29 \%$ & 0 & 0 & 0 \\
\hline LeBrun et al. [11] & 57 & 8 & $50.00 \%$ & 49 & $2.04 \%$ & 2 & 2 & 0 \\
\hline Macey et al. [25] & 73 & 10 & $20.00 \%$ & 63 & $\mathrm{a}$ & 3 & 0 & 3 \\
\hline Mamarelis et al. [26] & 31 & 8 & $37.50 \%$ & 23 & $4.35 \%$ & 6 & 3 & 3 \\
\hline Maniscalco et al. [27] & 121 & 32 & $43.75 \%$ & 89 & $3.37 \%$ & 0 & 0 & 0 \\
\hline Munoz et al. [28] & 124 & 15 & $13.33 \%$ & 109 & $2.75 \%$ & 12 & 8 & 4 \\
\hline Narang et al. [29] & 682 & 86 & $34.88 \%$ & 596 & $6.04 \%$ & 0 & 0 & 0 \\
\hline Rasidovic et al. [30] & 391 & 109 & a & 282 & $\mathrm{a}$ & 10 & 5 & 5 \\
\hline Thakrar et al. [31] & 43 & 13 & $38.46 \%$ & 30 & $6.67 \%$ & 0 & 0 & 0 \\
\hline Ward et al. [32] & 127 & 45 & $\mathrm{a}$ & 82 & $\mathrm{a}$ & 5 & 1 & 4 \\
\hline Totals & 2927 & 620 & & 2307 & & 71 & 39 & 32 \\
\hline
\end{tabular}

COVID-19 Coronavirus Disease of 2019

${ }^{\mathrm{a}}$ Group-specific mortality unspecified

Fig. 2 Forest plot showing postoperative CPHFP mortality in the short-term stratified by screening protocol
Study

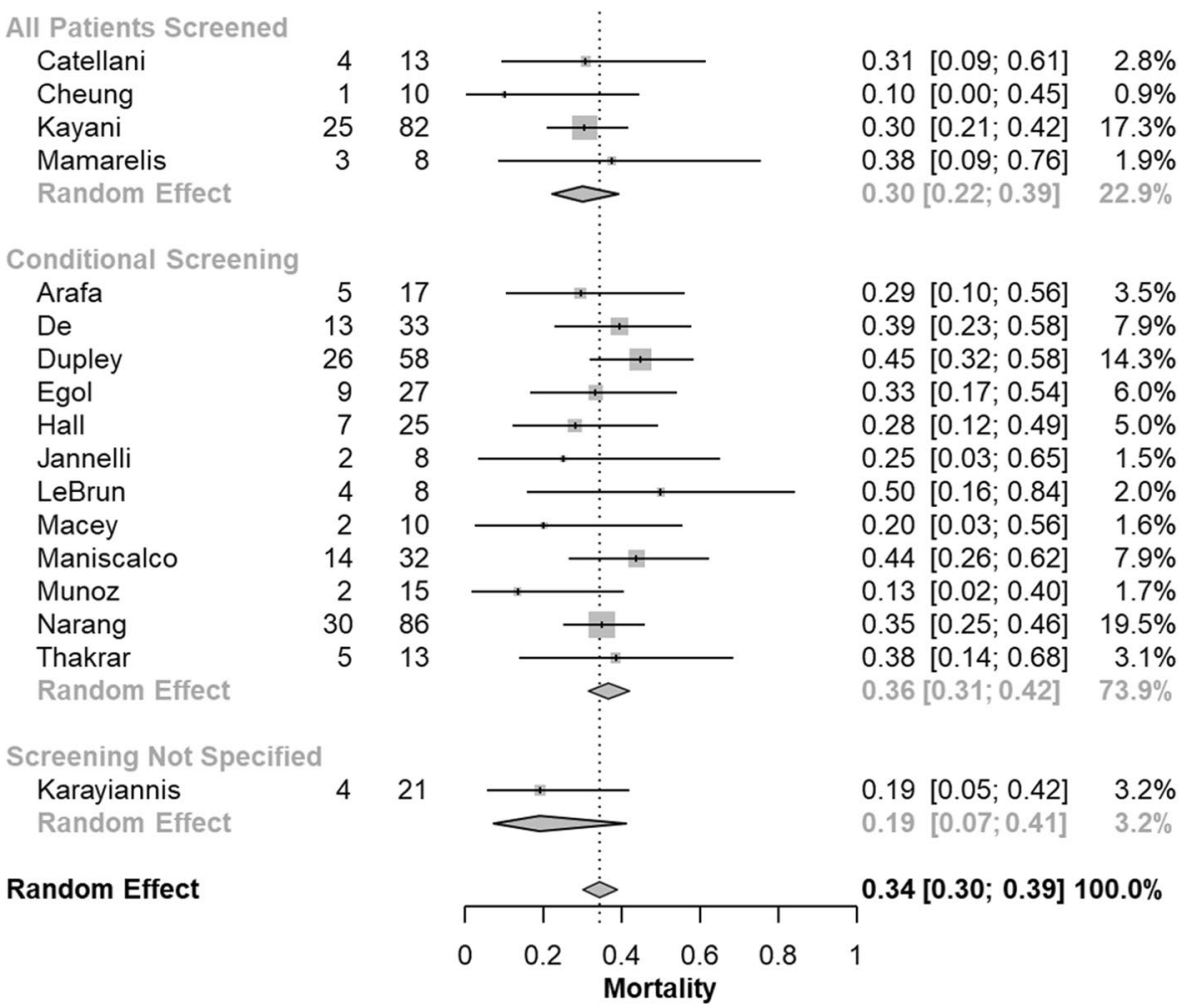


Fig. 3 Forest plot comparing postoperative CPHFP mortality to postoperative COVID-negative hip fracture patient mortality in the short-term stratified by screening protocol

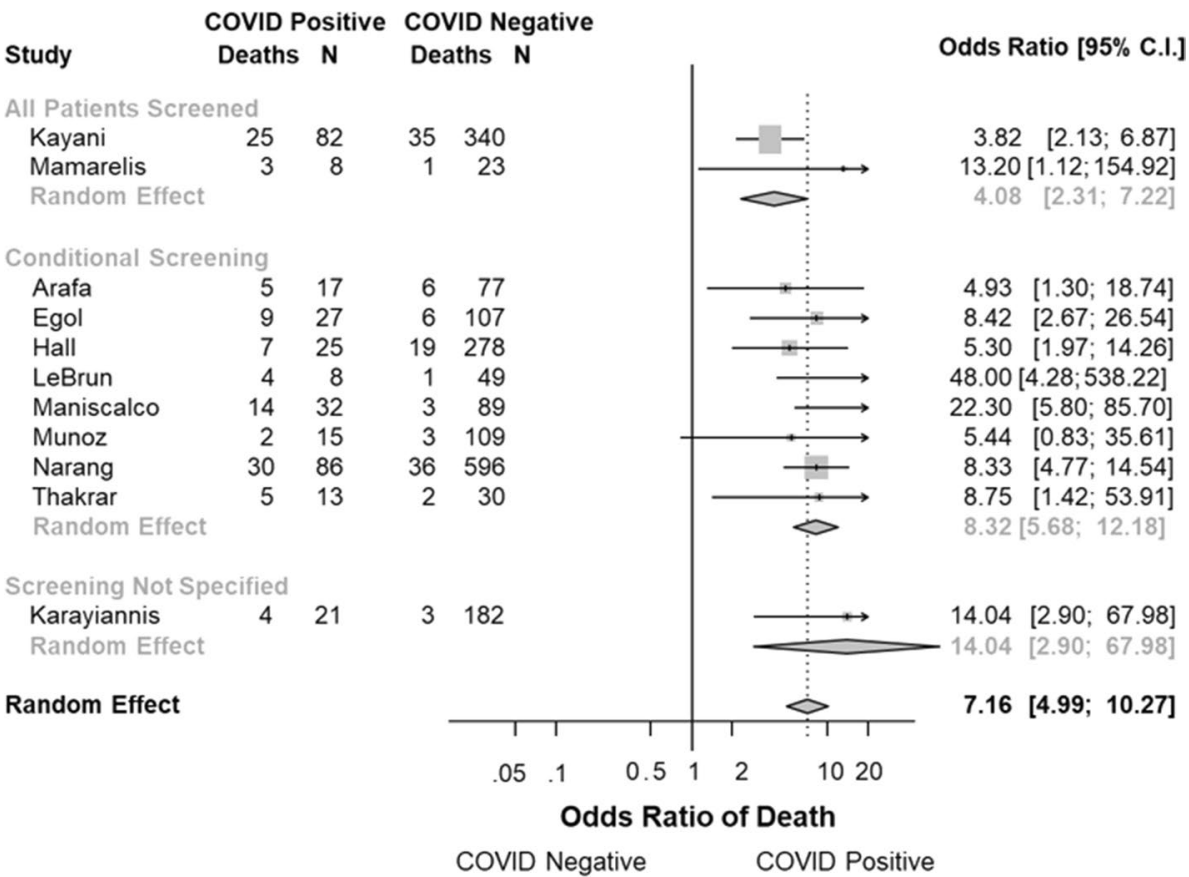

association between COVID-19 positive status and shortterm mortality in studies screening patients conditionally $(n=8 ; 223$ patients) produced an odds ratio of 8.32 (95\% C.I., 5.68-12.18), which was higher than the odds ratio produced by studies that screened all patients $(n=2 ; 90$ patients) of 4.08 (95\% C.I., 2.31-7.22). This difference was statistically significant $(P=0.04)$.

There was no evidence of publication bias upon review of the funnel plots; though for short-term mortality, there were a few observations with higher mortality and larger standard error that were not completely balanced (Fig. 4). In
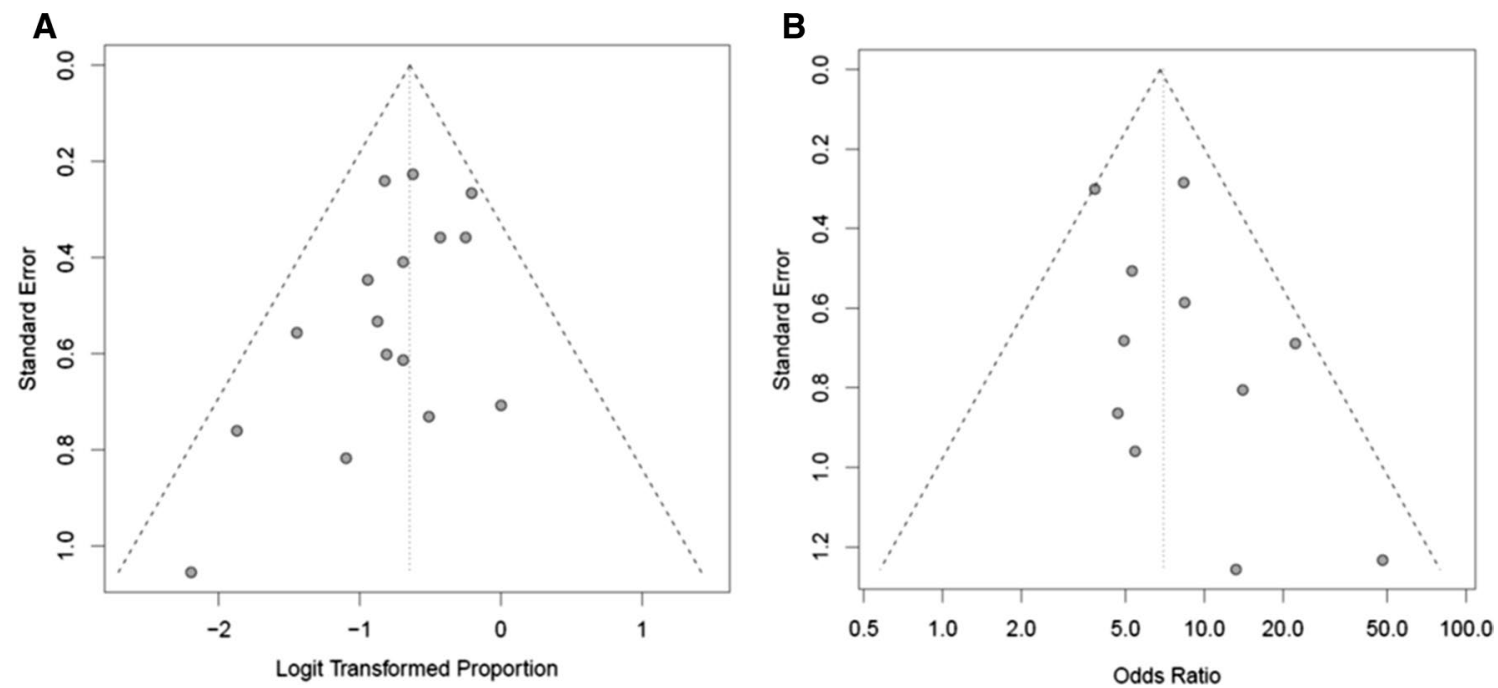

the two studies excluded from all meta-analyses, we could not extract discrete mortality rates for operatively managed CPHFPs [30, 32]. The two studies reported overall CPHFP mortality rates of 32.5 and $37.0 \%$ [30, 32].

\section{Discussion}

Our systematic review and meta-analysis of 17 studies ( $n=466$ operative CPHFPs) revealed a sevenfold increase in 30-day postoperative mortality rate of CPHFPs compared

Fig. 4 Funnel plots a logit transformed proportion and $\mathbf{b}$ odds ratio 
to operative COVID-negative hip fracture contemporary control. Overall, the short-term mortality rate of operative CPHFPs was approximately $34 \%$. To our knowledge, the present review is the first to report CPHFP postoperative mortality by COVID-19 screening protocol. We are also the first to report on the association between COVID-19 positive status and short-term hip fracture mortality with contemporary control based on screening protocol. We attempted this to make our observations more germane to the prognosis, counseling, and management of CPHFPs. We also believe this sub-analysis to be the first attempt to objectively investigate the association between degree of CPHFP symptomatology and risk of mortality, albeit an indirect investigation with more research required. Finally, we attempted to mitigate the effect of outliers on our data set by excluding studies reporting $<10$ CPHFPs, and the funnel plots generated represent no obvious concern for publication bias.

The present review has limitations. First, although we identified 19 clinical studies analyzing $\geq 10$ CPHFPs, mortality rates among operatively managed CPHFPs ranged from 10.0 to $50.0 \%$ [7-11, 19-32]. Only three studies reported overall mortality rates less than $20.0 \%$ [21, 24, 28]. Cheung et al. [21] reported on a 10-patient cohort of CPHFPs, eight of whom were asymptomatic on presentation. They reported an inpatient mortality rate of only $10.0 \%$, remarking that the mild nature of presentation could have contributed to their low mortality rate [21]. Concerning Munoz et al. [28], their follow-up period was shorter than most included studies at 14 days. Additionally, the mortality rates for the COVID-negative hip fracture controls in the Munoz et al. [28] and Karayiannis et al. [24] studies were two of the three lowest in this review, suggesting potentially broad confounding factors at play. Second, our data set exhibited a large range of sample sizes, though most were relatively small, particularly in some stratified analyses. Additionally, an inordinate number of patients was captured from studies in the United Kingdom (353/466 operative CPHFPs), limiting external validity. Third, designating some studies as having "conditional" screening protocols creates a heterogeneous group with wide-ranging protocols. Fourth, some operatively managed patients included in the CPHFP group were "presumed positive," or those patients who were deemed COVID-positive on the basis on clinical judgment despite absent screening results. While a potential confounder, we reasoned that counseling a "presumed positive" hip fracture patient as a CPHFP would be more appropriate than counseling them as COVID-negative hip fracture patient if indeed symptomatology is a key outcome determinant. Fifth, as previously stated, most of the currently available literature is retrospective and characterized by broad heterogeneity in terms of patient treatment, data collection, and data reporting. One data category to be noted is follow-up period. Among the patients included in this review, 78/466 operative CPHFPs were followed for less than 30 days. This may have contributed to underestimated mortality rate reporting. On the other hand, 58/466 operative CPHFPs patients included in this study were followed for 45 days. No trial or prospective data are available, and it is likely that the studies published thus far, and included in this review, will be the best available data to help guide future studies on this subject.

Nonetheless, our findings support a significantly higher early mortality with CPHFPs. Historical 30-day postoperative mortality rates for hip fractures in the elderly range from 5 to $7 \%$, rising to $15.6 \%$ in nonagenarians and $20.7 \%$ in centenarians [33]. Even one-year mortality rates for hip fracture patients are estimated to be lower than our findings at 30\% [21]. Moreover, the CPHFP mortality rates we observed are worse than those of COVID-19 patients with no reported comorbidities (0.9\% mortality), COVID-19 patients overall (2.3\% mortality), or COVID-19 patients aged $\geq 80$ years (up to $30 \%$ mortality) [12]. Our findings corroborate those reported in a recent, smaller meta-analysis wherein the authors found an early postoperative CPHFP mortality rate of $32.6 \%$ in a sample of 365 patients [12]. The authors noted CPHFP mortality rates are more severe than those associated with hip fracture or COVID-19 alone, concluding that COVID-19 may exhibit an effect modification on the risk of mortality in hip fracture patients [12].

Our analysis of CPHFP mortality did not identify significant differences based upon screening protocols. It should be noted that, should a significant difference actually exist, our small sample size may have been underpowered for detection. Among the 11 studies that reported a control group of contemporary COVID-negative hip fracture patients, CPHFPs demonstrated an odds ratio of mortality of 7.16 (95\% C.I., 4.99-10.27). This result is similar to previous meta-analyses, which have reported relative risk ratios for CPHFPs versus contemporary COVID-negative hip fracture patients of 7.45 and 5.66 [2, 12]. Interestingly, when stratified by screening protocol, the odds ratios of mortality among studies that screened all patients $(n=2$; combined sample size of 90$)$ and those that screened patients conditionally $(n=8$; combined sample size of 223$)$ were 4.08 (95\% C.I., 2.31-7.22) and 8.32 (95\% C.I., 5.68-12.18), respectively. This difference was statistically significant $(P=0.04)$. This observation lends credence to the theory that CPHFPs who are asymptomatic or minimally symptomatic of COVID-19 on admission may demonstrate lower rates of mortality compared to more severely symptomatic patients. If this were true, then capturing and grouping asymptomatic or minimally symptomatic CPHFPs with more symptomatic CPHFPs (as in studies that screened all patients) would decrease the overall mortality rate of the CPHFP group, as we have potentially observed. Indeed, some individual findings among studies included suggest potentially lower rates 
of mortality among asymptomatic CPHFPs. As previously stated, Cheung et al. [21] reported a $10.0 \%$ mortality rate among 10 CPHFPs, eight of whom were asymptomatic on presentation. Conversely, Maniscalco et al. [27] reported nine deaths among 19 CPHFPs who were symptomatic on admission versus two asymptomatic CPHFPs who both survived through follow-up.

One potential reason underlying the poor CPHFP outcomes we report may be the overlapping pathophysiological mechanisms between orthopedic injury and SARS-CoV-2 infection [13-15]. SARS-CoV-2 infection causes an uncontrolled immune response leading to cytokine release syndrome (CRS) and potentially acute respiratory distress syndrome [34-36]. These circumstances can be exacerbated by fracture itself and any additional surgical insult, the latter of which can be a detrimental "second hit" $[13,37]$. If this is the case, we might expect COVID-positive patients with other orthopedic injuries to also tolerate surgery poorly, and there is some literature to support this theory. For example, the COVIDsurg Collaborative, an observational cohort study of 1128 COVID-19 patients undergoing surgery of any kind (26.8\% were orthopedic), reported an overall 30-day mortality rate of $23.8 \%$ (28.8\% for orthopedic surgeries) [38]. In a matched cohort study of 41 COVID-19 patients undergoing different types of surgeries (53.7\% underwent orthopedic surgeries), Doglietto et al. [39] reported a 30-day mortality of 19.5 versus $2.44 \%$ in the control group (COVID-negative surgical patients). In sum, CPHFPs present at the intersection of multiple jeopardizing factors: they face three potentiators of CRS (COVID-19, fracture, and orthopedic surgery) $[13,37]$. Additionally, they are an elderly population with a high comorbidity burden [5-11, 13, 31, 37].

While it is reasonable to regard CPHFPs as a susceptible group, questions still remain. For example, while there is some evidence in the present review that the severity of COVID-19 symptoms on admission may have an association with mortality, this information was rarely reported. Thus, we are unable to draw strong conclusions. Questions also remain about outcomes aside from mortality, such as rates of venous thromboembolism and optimal prophylactic regimens. Given the continued prevalence of hip fractures amid the pandemic, we believe that undertaking more prospective research concerning CPHFP surgical outcomes could furnish relevant data and insight.

\section{Conclusions}

Our findings suggest that COVID-positive hip fracture patients have a sevenfold increased risk (34\%) of 30-day postoperative mortality. Screening all patients led to decreased mortality risk. This suggests mortality prognostication should consider how COVID-19 infection was identified as asymptomatic patients may fare slightly better. Undertaking larger scale prospective studies could provide valuable data for evidence-based management of this population.

Supplementary Information The online version contains supplementary material available at https://doi.org/10.1007/s00590-022-03228-9.

Funding No funding was received for this manuscript.

\section{Declarations}

Conflict of interest The authors have no competing interests to declare that are relevant to the content of this article. Postoperative Mortality in the COVID-Positive Hip Fracture Patient, a Systematic Review and Meta-Analysis.

\section{References}

1. Dong E, Du H, Gardner L (2020) An interactive web-based dashboard to track COVID-19 in real time. Lancet Infect Dis 20:533534. https://doi.org/10.1016/S1473-3099(20)30120-1

2. Lim MA, Pranata R (2020) Coronavirus disease 2019 (COVID19) markedly increased mortality in patients with hip fracture - a systematic review and meta-analysis. J Clin Orthop Trauma. https://doi.org/10.1016/j.jcot.2020.09.015

3. Centers for Disease Control and Prevention. Hip Fractures Among Older Adults. Accessed October 6, 2020, https://www.cdc.gov/ homeandrecreationalsafety/falls/adulthipfx.html\#: :text=Each\% 20year\%20over\%20300\%2C000\%20older,2\%20usually $\% 20$ by $\%$ 20 falling $\% 20$ sideways.\&text $=$ Women $\% 20$ experience $\% 20$ three $\%$ 2Dquarters\%20of,fall\%20more\%20often\%20than $\% 20$ men.

4. Kumar Jain V, Lal H, Kumar Patralekh M, Vaishya R (2020) Fracture management during COVID-19 pandemic: A systematic review. J Clin Orthop Trauma 11:S431-S441. https://doi.org/10. 1016/j.jcot.2020.06.035

5. Cheng B, Jiang T, Zhang L, et al (2020) Clinical characteristics of pregnant women with coronavirus disease 2019 in Wuhan, China. Open Forum Infect Dis 7:ofaa294. https://doi.org/10.1093/ofid/ ofaa294

6. Deng Y, Liu W, Liu K et al (2020) Clinical characteristics of fatal and recovered cases of coronavirus disease 2019 in Wuhan, China: a retrospective study. Chin Med J (Engl) 133:1261-1267. https:// doi.org/10.1097/CM9.0000000000000824

7. Dupley L, Oputa TJ, Bourne JT, North West CNOFSG (2020) 30-day mortality for fractured neck of femur patients with concurrent COVID-19 infection. Eur J Orthop Surg Traumatol. https:// doi.org/10.1007/s00590-020-02778-0

8. Egol KA, Konda SR, Bird ML et al (2020) Increased mortality and major complications in hip fracture care during the COVID19 Pandemic: a New York City perspective. J Orthop Trauma 34:395-402. https://doi.org/10.1097/BOT.0000000000001845

9. Hall AJ, Clement ND, Farrow L, et al (2020) IMPACT-Scot report on COVID-19 and hip fractures. Bone Joint J 102-B:1219-1228. https://doi.org/10.1302/0301-620X.102B9.BJJ-2020-1100.R1

10. Kayani B, Onochie E, Patil V, et al (2020) Infographic: the effects of COVID-19 on perioperative morbidity and mortality in patients with hip fractures. Bone Joint J 102-B:1279-1280. https://doi.org/ 10.1302/0301-620X.102B10.BJJ-2020-1774 
11. LeBrun DG, Konnaris MA, Ghahramani GC et al (2020) Hip fracture outcomes during the COVID-19 Pandemic: early results from New York. J Orthop Trauma 34:403-410. https://doi.org/10. 1097/BOT.0000000000001849

12. Wang KC, Xiao R, Cheung ZB, Barbera JP, Forsh DA (2020) Early mortality after hip fracture surgery in COVID-19 patients: a systematic review and meta-analysis. J Orthop 22:584-591. https://doi.org/10.1016/j.jor.2020.11.012

13. Puzzitiello RN, Pagani NR, Moverman MA, Moon AS, Menendez ME, Ryan SP (2020) Inflammatory and coagulative considerations for the management of orthopaedic trauma patients with COVID19: a review of the current evidence and our surgical experience. $J$ Orthop Trauma 34:389-394. https://doi.org/10.1097/BOT.00000 00000001842

14. Tay MZ, Poh CM, Renia L, MacAry PA, Ng LFP (2020) The trinity of COVID-19: immunity, inflammation and intervention. Nat Rev Immunol 20:363-374. https://doi.org/10.1038/ s41577-020-0311-8

15. Tufan A, Avanoglu Guler A, Matucci-Cerinic M (2020) COVID19 , immune system response, hyperinflammation and repurposing antirheumatic drugs. Turk J Med Sci 50:620-632. https://doi.org/ 10.3906/sag-2004-168

16. Moher D, Liberati A, Tetzlaff J, Altman DG, Group P (2009) Preferred reporting items for systematic reviews and meta-analyses: the PRISMA statement. PLoS Med 6:e1000097. https://doi.org/ 10.1371/journal.pmed.1000097

17. Slim K, Nini E, Forestier D, Kwiatkowski F, Panis Y, Chipponi J (2003) Methodological index for non-randomized studies (minors): development and validation of a new instrument. ANZ J Surg 73:712-716. https://doi.org/10.1046/j.1445-2197.2003. 02748.x

18. Meinberg EG, Agel J, Roberts CS, Karam MD, Kellam JF (2018) Fracture and dislocation classification compendium-2018. J Orthop Trauma 32(Suppl 1):S1-S170. https://doi.org/10.1097/ BOT.0000000000001063

19. Arafa M, Nesar S, Abu-Jabeh H, Jayme MOR, Kalairajah Y (2020) COVID-19 pandemic and hip fractures: impact and lessons learned. Bone Jt Open 1:530-540. https://doi.org/10.1302/ 2633-1462.19.BJO-2020-0116.R1

20. Catellani F, Coscione A, D'Ambrosi R, Usai L, Roscitano C, Fiorentino G (2020) Treatment of proximal femoral fragility fractures in patients with COVID-19 during the SARS-CoV-2 outbreak in Northern Italy. J Bone Joint Surg Am 102:e58. https:// doi.org/10.2106/JBJS.20.00617

21. Cheung ZB, Forsh DA (2020) Early outcomes after hip fracture surgery in COVID-19 patients in New York City. J Orthop 21:291-296. https://doi.org/10.1016/j.jor.2020.06.003

22. De C, Wignall A, Giannoudis V, et al (2020) Peri-operative outcomes and predictors of mortality in COVID-19 positive patients with hip fractures: a multicentre study in the UK. Indian J Orthop:1-11. https://doi.org/10.1007/s43465-020-00272-7

23. Jannelli E, Castelli A, Ferranti Calderoni E et al (2020) Fractures in patients with COVID-19 infection: early prognosis and management. A case series of 20 patients in a single institution in Lombardy. Northern Italy J Orthop Trauma 34:e389-e397. https:// doi.org/10.1097/BOT.0000000000001905

24. Karayiannis PN, Roberts V, Cassidy R et al (2020) 30-day mortality following trauma and orthopaedic surgery during the peak of the COVID-19 pandemic: a multicentre regional analysis of 484 patients. Bone Jt Open 1:392-397. https://doi.org/10.1302/26331462.17.BJO-2020-0075.R1

25. Macey ARM, Butler J, Martin SC, Tan TY, Leach WJ, Jamal B (2020) 30-day outcomes in hip fracture patients during the COVID-19 pandemic compared to the preceding year. Bone Jt Open 1:415-419. https://doi.org/10.1302/2633-1462.17.BJO2020-0077.R1
26. Mamarelis G, Oduoza U, Chekuri R, Estfan R, Greer T (2020) Mortality in patients with proximal femoral fracture during the COVID-19 Pandemic: A U.K. Hospital's Experience. JB JS Open Access 510.2106/JBJS.OA.20.00086

27. Maniscalco P, Poggiali E, Quattrini F, et al (2020) Proximal femur fractures in COVID-19 emergency: the experience of two Orthopedics and Traumatology Departments in the first eight weeks of the Italian epidemic. Acta Biomed 91:89-96. https://doi.org/10. 23750/abm.v91i2.9636

28. Munoz Vives JM, Jornet-Gibert M, Camara-Cabrera J et al (2020) Mortality rates of patients with proximal femoral fracture in a worldwide pandemic: preliminary results of the Spanish HIPCOVID Observational Study. J Bone Joint Surg Am 102:e69. https://doi.org/10.2106/JBJS.20.00686

29. Narang A, Chan G, Aframian A et al (2020) Thirty-day mortality following surgical management of hip fractures during the COVID-19 pandemic: findings from a prospective multi-centre UK study. Int Orthop. https://doi.org/10.1007/ s00264-020-04739-y

30. Rasidovic D, Ahmed I, Thomas $C$ et al (2020) Impact of COVID19 on clinical outcomes for patients with fractured hip: a multicentre observational cohort study. Bone Jt Open 1:697-705. https://doi.org/10.1302/2633-1462.111.BJO-2020-0132.R1

31. Thakrar A, Chui K, Kapoor A, Hambidge J (2020) 30-day mortality rate of patients with hip fractures during the COVID-19 Pandemic: a single centre prospective study in the United Kingdom. J Orthop Trauma. https://doi.org/10.1097/BOT.0000000000001889

32. Ward AE, Tadross D, Wells F et al (2020) The impact of COVID19 on morbidity and mortality in neck of femur fracture patients: a prospective case-control cohort study. Bone Jt Open 1:669-675. https://doi.org/10.1302/2633-1462.111.BJO-2020-0141.R1

33. Barcelo M, Francia E, Romero C, Ruiz D, Casademont J, Torres $\mathrm{OH}$ (2018) Hip fractures in the oldest old. Comparative study of centenarians and nonagenarians and mortality risk factors. Injury 49:2198-2202. https://doi.org/10.1016/j.injury.2018.09.043

34. Risitano AM, Mastellos DC, Huber-Lang M et al (2020) Complement as a target in COVID-19? Nat Rev Immunol 20:343-344. https://doi.org/10.1038/s41577-020-0320-7

35. Ruan Q, Yang K, Wang W, Jiang L, Song J (2020) Clinical predictors of mortality due to COVID-19 based on an analysis of data of 150 patients from Wuhan, China. Intensive Care Med 46:846-848. https://doi.org/10.1007/s00134-020-05991-x

36. Mehta P, McAuley DF, Brown M et al (2020) COVID-19: consider cytokine storm syndromes and immunosuppression. Lancet 395:1033-1034. https://doi.org/10.1016/S0140-6736(20)30628-0

37. Lasanianos NG, Kanakaris NK, Dimitriou R, Pape HC, Giannoudis PV (2011) Second hit phenomenon: existing evidence of clinical implications. Injury 42:617-629. https://doi.org/10. 1016/j.injury.2011.02.011

38. Collaborative CO (2020) Mortality and pulmonary complications in patients undergoing surgery with perioperative SARS-CoV-2 infection: an international cohort study. Lancet 396:27-38. https:// doi.org/10.1016/S0140-6736(20)31182-X

39. Doglietto F, Vezzoli M, Gheza F et al (2020) Factors associated with surgical mortality and complications among patients with and without Coronavirus Disease 2019 (COVID-19) in Italy. JAMA Surg. https://doi.org/10.1001/jamasurg.2020.2713

Publisher's Note Springer Nature remains neutral with regard to jurisdictional claims in published maps and institutional affiliations. 